\section{Câncer em mulheres idosas das regiões Sul e Sudeste do Brasil: Evolução da mortalidade no período 1980 - 2005}

\section{Cancer in elderly women in the South and Southeast regions of Brazil: mortality trends in the 1980- 2005 period}

\author{
Denise Vianna Basílio' \\ Inês Echenique Mattos ${ }^{2}$ \\ ${ }^{1}$ Casa Gerontológica da Aeronáutica Brigadeiro Eduardo Gomes, Programa de \\ Pós-Graduação em Saúde Pública e Meio Ambiente da ENSP/FIOCRUZ \\ ${ }^{2}$ Departamento de Epidemiologia da Escola Nacional de Saúde Pública/ \\ FIOCRUZ
}

Correspondência: Inês E. Mattos. Departamento de Epidemiologia, Escola Nacional de Saúde Pública - FIOCRUZ. Rua Leopoldo Bulhões 1480 - Manguinhos. CEP 21041-210 Rio de Janeiro, RJ. E-mail: imattos@ensp.fiocruz.br

\section{Resumo}

Introdução: Com o envelhecimento progressivo da população, as neoplasias se constituíram em importante causa de morbimortalidade no Brasil. As regiões Sul e Sudeste apresentam as maiores taxas de mortalidade por câncer e abrigam os maiores contingentes de população idosa. Objetivo: Analisar a tendência da mortalidade por localizações selecionadas de câncer na população feminina de 60 anos ou mais das regiões Sul e Sudeste do Brasil, no período 1980-2005. Metodologia: Os dados anuais da população residente e de mortalidade por câncer em geral e por câncer de esôfago, estômago, cólon/reto, pâncreas, pulmão, mama e colo de útero foram obtidos no DATASUS. A análise da tendência das taxas de mortalidade foi efetuada para três faixas etárias, a partir de 60 anos, através de modelos de regressão. Resultados: Verificouse tendência significativa de crescimento da mortalidade por câncer de cólon/reto, pâncreas, pulmão e mama nas três faixas etárias nas duas regiões. O câncer de estômago apresentou tendência significativa de decréscimo em todas as faixas etárias nas regiões Sul e Sudeste, enquanto para câncer de esôfago observou-se tendência significativa de declínio somente no Sudeste. Conclusões: Os padrões de mortalidade por câncer de mulheres idosas do Sul e Sudeste do Brasil acompanham tendências mundiais, tendo ocorrido importantes variações no período de estudo. Mecanismos envolvidos no comportamento da mortalidade em idosos, em grande parte ainda desconhecidos, assim como diferenças regionais e entre as gerações na prevalência de fatores de risco e proteção para câncer, poderiam explicar parcialmente os padrões observados. São necessários estudos que aprofundem a análise da mortalidade por câncer nesse grupo populacional.

Palavras-chave: Mortalidade. Câncer. Mulheres idosas. 


\section{Abstract}

Introduction: With the gradual aging of the Brazilian population, cancer has gained an increasingly important role as a cause of morbidity and mortality. The South and Southeast regions of Brazil present the highest rates of cancer mortality and are inhabited by the greatest number of elderly people. Objective: To analyze patterns of mortality for selected cancer sites in the female population of 60 years of age or older in the South and Southeast regions of Brazil, in the 1980-2005 period. Method: Population and mortality data for all cancers and for esophageal, gastric, colon/ rectal, pancreas, breast, and uterine cervix cancer, by year of the study period, were obtained from the Ministry of Health/DATASUS. Regression models were estimated to analyze trends in mortality rates for three age groups: 60-69 years, 70-79 years, and 80 years or more. Results: Mortality rates for colon-rectal, pancreas, lung and breast cancer showed significantly rising trends in all age groups in both regions. A significant decreasing trend was observed for gastric cancer in the three age groups, both in the South and in the Southeast region, while significantly decreasing trends were observed for esophageal cancer only in the Southeast region Conclusions: Patterns of cancer mortality in elderly women of the South and Southeast regions of Brazil follow world-wide trends and important changes in these trends occurred during the study period. Mechanisms involved in the behavior of cancer mortality in aging individuals, still unknown to a large extent, and regional and generational differences in the prevalence of risk and protective factors for cancer could partially explain some of the patterns observed. Further studies on cancer mortality in aging individuals are needed to extend our present knowledge.

Keywords: Mortality. Cancer. Elderly women.

\section{Introdução}

À medida que aumenta a esperança de vida e cresce o número de idosos, a exposição constante a fatores de risco para doenças crônico-degenerativas faz com que se tornem mais freqüentes as complicações de saúde por esses agravos. Segundo projeções realizadas pela OMS, no ano de 2025 o Brasil será o sexto país do mundo com maior número de pessoas com 60 anos ou mais ${ }^{1}$. Quanto mais idoso for o grupo populacional estudado, maior a proporção de mulheres entre estes. Em 1996, 54,4\% da população idosa do Brasil pertenciam ao sexo feminino ${ }^{2}$.

Nesse contexto de envelhecimento progressivo da população e crescimento das doenças crônicas, o câncer tem-se revelado importante causa de morbimortalidade em indivíduos com 60 ou mais anos, nos diferentes países do mundo. No Brasil, no período 1980-2000, as taxas de mortalidade por câncer em indivíduos idosos apresentaram incremento gradativo, fazendo desta enfermidade a segunda causa de morte nesse grupo populacional, onde predominam, no sexo feminino, os óbitos por câncer de mama, pulmão e estômago ${ }^{3}$.

Importantes diferenças na sobrevida de câncer entre adultos jovens e idosos têm sido apontadas na literatura, com desvantagem mais acentuada para o sexo feminino ${ }^{4}$. As regiões Sul e Sudeste caracterizam-se por apresentar as maiores taxas de mortalidade por câncer do país ${ }^{5}$, e abrigam os maiores contingentes de população idosa do Brasil ${ }^{6}$.

Considerando a forte associação entre idade e risco de câncer, torna-se interessante analisar o comportamento da mortalidade por câncer em diferentes grupos etários da população feminina idosa nessas duas regiões.

O objetivo deste estudo é analisar a tendência das taxas de mortalidade por localizações selecionadas de câncer na população feminina de 60 anos ou mais das regiões Sul e Sudeste do Brasil, no período de 1980 a 2005. 


\section{Material e métodos}

Foi realizado um estudo descritivo para analisar a tendência da mortalidade por localizações selecionadas de câncer em mulheres de 60 ou mais anos de idade, residentes nas regiões Sul e Sudeste, no período de 1980 a 2005.

Os óbitos por todas as causas de câncer e pelas localizações selecionadas (esôfago, estômago, cólon/reto, pâncreas, mama e colo de útero), ocorridos no período 1980-2005, em mulheres de 60 ou mais anos de idade, residentes nas regiões Sul e Sudeste do Brasil, foram obtidos no DATASUS ${ }^{6}$, através das Listas Básicas de Tabulação da Classificação Internacional de Doenças (CID-BR), adaptadas à realidade brasileira pela Secretaria de Vigilância em Saúde. Os óbitos ocorridos no período 1980 a 1995 foram retirados da CID-BR 9, baseada na 9a revisão da Classificação Internacional de Doenças (CID 9), então vigente, e para aqueles ocorridos no período 1996-2005 foi utilizada a CID-BR 10, originada da CID-10. As informações sobre a população feminina residente nessas regiões, por faixa etária (60-69; 70-79; 80 anos e mais), foram obtidas também no DATASUS e têm como base os censos demográficos de 1980 e 2000 e estimativas populacionais para os anos intercensitários do Instituto Brasileiro de Geografia e Estatística.

Taxas médias de mortalidade por câncer em geral e pelas localizações estudadas nas regiões Sul e Sudeste, segundo três faixas etárias (60-69 anos; 70-79 anos e 80 ou mais anos de idade), foram calculadas para os triênios 1980-82, 1991-93 e 2003-05. Os percentuais de variação das taxas de mortalidade foram analisados comparando-se os valores do primeiro e do último triênio, através da fórmula: [(taxa do triênio 3 - taxa do triênio 1) / taxa do triênio 1 x 100].

Foram calculadas as taxas anuais de mortalidade para as diferentes localizações de câncer nas duas regiões, segundo as três faixas etárias estudadas, as quais foram utilizadas na análise da tendência da mortalidade, através da estimativa de modelos de regressão. Para fins dessa análise, as taxas de mortalidade por cada localização de neoplasia em cada faixa etária e região de estudo (y) foram consideradas como variável dependente e, como variável independente (x), os anos do período de estudo. Foram testados modelos de regressão linear simples $\left(y=\beta_{0}+\beta_{1} x\right)$, segundo grau $\left(y=\beta_{0}+\right.$ $\left.\beta_{1} x+\beta_{2} x^{2}\right)$ e terceiro graus $\left(y=\beta_{0}+\beta_{1} x+\beta_{2} x^{2}+\right.$ $\beta_{3} x^{3}$ ). Visando evitar a correlação serial entre os termos das equações de regressão, optamos por centralizar a variável ano. Como critérios para a escolha do melhor modelo, foram considerados o nível de significância estatística e a análise dos resíduos. Quando mais de um modelo satisfazia os critérios de escolha, selecionou-se o mais simples.

Os softwares Excel 2003 e SPSS versão 11.0 foram utilizados para a análise dos dados.

Esse estudo foi aprovado pelo Comitê de Ética em Pesquisa da Escola Nacional de Saúde Pública da Fundação Oswaldo Cruz.

Os autores, através de documentação assinada e enviada a RBE, declaram não existir nenhum tipo de conflito de interesses.

\section{Resultados}

As taxas de mortalidade por câncer em geral e por localizações selecionadas em mulheres de 60 anos ou mais nas regiões Sul e Sudeste e os respectivos percentuais de variação estão na Tabela 1. No período de estudo, observou-se variação percentual negativa das taxas de mortalidade por câncer na região Sudeste e variação percentual positiva na região Sul no grupo etário de 60-69 anos. Para o grupo de 70-79 anos, observouse variação percentual positiva na região Sul, enquanto na região Sudeste as taxas de mortalidade mantiveram-se praticamente constantes. No grupo de 80 ou mais anos de idade, as taxas da região Sul apresentaram maior magnitude que as do Sudeste, tendo ambas regiões apresentado variação percentual positiva entre 1980 e 2005. A região Sul apresentou, para todos os grupos etários, taxas de maior magnitude para câncer de esôfago, pâncreas e pulmão. Ambas as regiões apresentaram variações percentuais negativas para câncer de esôfago e estômago 
Tabela 1 - Taxas de mortalidade por câncer em geral e localizações de câncer selecionadas em mulheres de 60 ou mais anos de idade por faixa etária e variação percentual no período 1980-2005 nas regiões Sul e Sudeste do Brasil.

Table 1 - Mortality rates for all cancers and for selected cancer sites in women aged 60 years or older by age group and percentage of variation in the 1980-2005 period in the South and Southeast regions of Brazil.

\begin{tabular}{|c|c|c|c|c|c|c|c|c|c|c|c|c|}
\hline \multirow{3}{*}{$\begin{array}{l}\text { Faixa etária } \\
\text { Localização }\end{array}$} & \multicolumn{4}{|c|}{$60-69$ anos } & \multicolumn{4}{|c|}{ 70-79 anos } & \multicolumn{4}{|c|}{80 anos e +} \\
\hline & \multicolumn{3}{|c|}{ Taxas de mortalidade } & \multirow[t]{2}{*}{ \% variação } & \multicolumn{3}{|c|}{ Taxas de mortalidade } & \multirow[t]{2}{*}{ \% variação } & \multicolumn{3}{|c|}{ Taxas de mortalidade } & \multirow[t]{2}{*}{ \% variação } \\
\hline & $1980-82$ & $1991-93$ & $2003-05$ & & $1980-82$ & $1991-93$ & $2003-05$ & & $1980-82$ & $1991-93$ & 2003-05 & \\
\hline \multicolumn{13}{|l|}{ Região Sul } \\
\hline Esôfago & 17,5 & 15,2 & 15,67 & $-10,2$ & 39,4 & 33,2 & 27,4 & $-29,7$ & 59,8 & 54,4 & 49,5 & $-17,2$ \\
\hline Estômago & 38,0 & 28,9 & 21,4 & $-43,4$ & 84,7 & 68,2 & 52,7 & $-37,7$ & 138,4 & 121,7 & 97,7 & $-29,4$ \\
\hline Cólon e reto & 22,6 & 24,0 & 31,9 & 41,2 & 59,1 & 59,3 & 65,4 & 10,5 & 110,3 & 112,4 & 148,4 & 34,6 \\
\hline Pâncreas & 15,4 & 17,1 & 20,3 & 31,6 & 28,5 & 38,8 & 45,1 & 58,5 & 44,0 & 58,0 & 92,4 & 110,0 \\
\hline Pulmão & 24,2 & 38,0 & 51,2 & 112,2 & 43,3 & 63,5 & 88,9 & 105,5 & 49,3 & 76,0 & 108,1 & 119,4 \\
\hline Mama & 39,5 & 49,3 & 49,7 & 25,7 & 48,2 & 67,7 & 78,5 & 63,0 & 76,2 & 105,9 & 130,5 & 71,1 \\
\hline Colo de útero & 15,2 & 18,3 & 19,9 & 30,8 & 21,2 & 23,0 & 20,9 & $-1,2$ & 21,1 & 23,5 & 24,0 & 9,8 \\
\hline Todas & 338,8 & 359,2 & 382,5 & 12,9 & 627,9 & 683,7 & 725,6 & 15,8 & 946,0 & 1049,4 & 1245,6 & 31,7 \\
\hline \multicolumn{13}{|l|}{ Região Sudeste } \\
\hline Esôfago & 9,8 & 8,7 & 7,4 & $-24,7$ & 22,2 & 15,0 & 14,7 & $-33,7$ & 28,2 & 26,3 & 24,2 & $-14,1$ \\
\hline Estômago & 43,7 & 30,4 & 21,4 & $-50,9$ & 99,8 & 68,7 & 50,1 & $-49,8$ & 157,3 & 132,3 & 95,7 & $-39,2$ \\
\hline Cólon e reto & 25,1 & 25,1 & 29,2 & 16,6 & 54,6 & 52,1 & 66,6 & 22,2 & 97,9 & 94,5 & 135,9 & 38,8 \\
\hline Pâncreas & 13,0 & 14,2 & 16,1 & 23,7 & 26,0 & 29,1 & 36,1 & 39,1 & 40,6 & 45,8 & 62,4 & 53,9 \\
\hline Pulmão & 23,5 & 27,6 & 33,8 & 44,2 & 39,7 & 49,0 & 60,7 & 52,9 & 49,1 & 64,9 & 85,5 & 74,0 \\
\hline Mama & 46,3 & 53,4 & 51,9 & 12,1 & 58,9 & 70,9 & 76,8 & 30,5 & 89,3 & 100,3 & 124,8 & 39,7 \\
\hline Colo de útero & 18,9 & 17,1 & 16,1 & $-14,8$ & 25,7 & 21,6 & 20,3 & $-21,1$ & 26,4 & 27,7 & 25,7 & $-2,5$ \\
\hline Todas & 346,7 & 342,8 & 333,6 & $-3,8$ & 627,3 & 608,5 & 628,7 & 0,2 & 927,4 & 968,2 & 1090,5 & 17,6 \\
\hline
\end{tabular}

e variações percentuais positivas para câncer de cólon e reto, pâncreas, pulmão e mama, em todas as faixas etárias.

A análise da tendência das taxas de mortalidade por câncer na região Sudeste não permitiu a identificação de modelos com significância estatística para as mulheres de 60-69 e 70-79 anos. Na região Sul, os modelos lineares selecionados mostraram tendência significativa de crescimento, com aumento de 2,47 óbitos por 100.000 mulheres/ano e coeficiente de determinação $\left(\mathrm{R}^{2}\right)$ de 0,61 entre as mulheres de 60-69 anos, tendo a taxa de mortalidade por câncer aumentado em média 4,29 óbitos por 100.000 mulheres de 70-79 anos $\left(R^{2}=0,54\right)$. Em relação à tendência das taxas de mortalidade do grupo de 80 anos ou mais, novamente foram selecionados modelos lineares e se observou incremento nas duas regiões, com aumento médio de 13,27 óbitos e 7,43 óbitos por 100.000 mulheres, respectivamente, nas regiões Sul $\left(R^{2}=0,59\right)$ e Sudeste $\left(R^{2}=0,59\right)$.

Na Figura 1, podemos observar a evolução das taxas de mortalidade por câncer de esôfago, estômago, cólon e reto e pâncreas na região Sudeste, entre 1980-2005. O modelo linear mostrou significância estatística nas três faixas etárias, tanto para câncer de esôfago como de estômago. Para mulheres entre 60-69 anos, as taxas de mortalidade para câncer de esôfago e estômago diminuíram em média 0,10 e 0,89 óbito por 100.000 mulheres, respectivamente, com o $\mathrm{R}^{2}$ de 0,42 e de 0,90 , enquanto no grupo de 70-79 anos, esta queda foi de 0,32 para esôfago $\left(\mathrm{R}^{2}=0,62\right)$ e de 2,11 para estômago $\left(\mathrm{R}^{2}=0,92\right)$, correspondendo a 0,31 e 2,83 por 100.000 mulheres de 80 anos e mais $\left(R^{2}=0,32\right.$ para esôfago e $R^{2}=0,85$ para estômago). Em contrapartida, foi observada tendência de incremento da mortalidade por câncer de cólon e reto e de pâncreas, nos três grupos etários, com o modelo linear apresentando significância estatística em todos os casos. Para câncer de cólon e reto, a cada ano, houve aumento de 0,25 óbito por 100.000 mulheres de 60-69 anos $\left(\mathrm{R}^{2}=0,60\right)$, de 0,57 óbito em mulheres de $70-79$ anos $\left(R^{2}=0,57\right) \mathrm{e}$ de 1,72 óbito em mulheres de 80 ou mais anos de idade $\left(R^{2}=0,65\right)$. As taxas de mortalidade por câncer de pâncreas aumentaram, a cada ano, em média, 0,15, 0,51 e 1,07 óbito por 


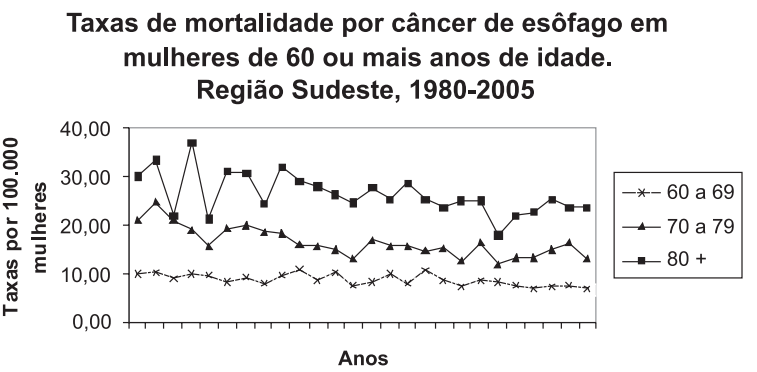

Taxas de mortalidade por câncer de cólon e reto em mulheres de 60 ou mais anos de idade Região Sudeste, 1980-2005

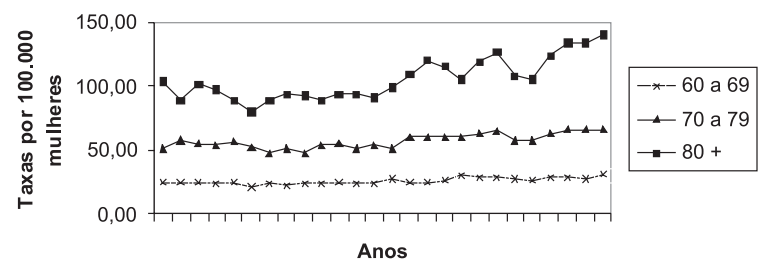

Taxas de mortalidade por câncer de estômago em mulheres de 60 ou mais anos de idade Região Sudeste 1980-2005

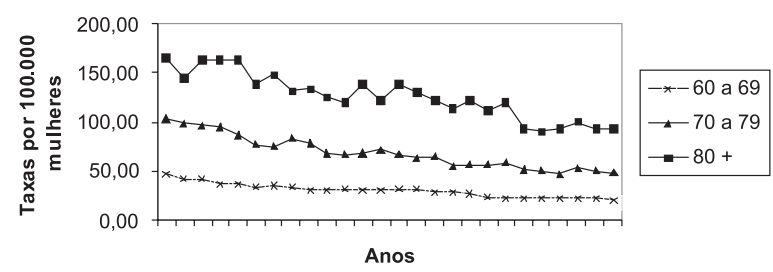

Taxas de mortalidade por câncer de pâncreas em mulheres de 60 ou mais anos de idade Região Sudeste 1980-2005

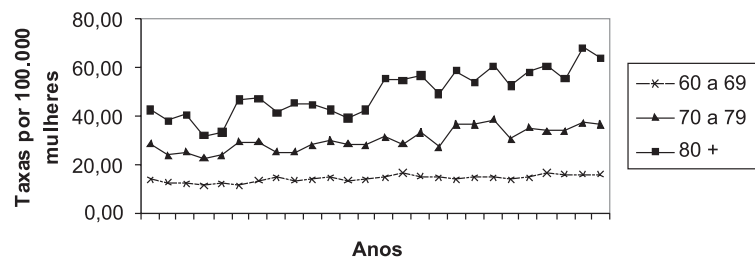

Figura 1 - Taxas de mortalidade por faixa etária (por 100.000) por câncer de esôfago, estômago, cólon e reto e pâncreas em mulheres de 60 ou mais anos de idade na região Sudeste do Brasil no período 1980-2005.

Figure 1 - Mortality rates per age group (per 100,000) for esophageal, gastric, colon/rectal and pancreatic cancers in women aged 60 or more in the Southeast region of Brazil in the 1980-2005 period.

100.000 mulheres, respectivamente para o grupo de 60-69 anos $\left(\mathrm{R}^{2}=0,61\right), 70-79$ anos $\left(\mathrm{R}^{2}\right.$ $=0,68)$ e 80 ou mais anos $\left(\mathrm{R}^{2}=0,75\right)$.

Na Figura 2 encontra-se a evolução das taxas de mortalidade dessas localizações de câncer na região Sul. Não foi possível identificar modelos com significância estatística na análise da tendência das taxas de mortalidade por câncer de esôfago, nos diferentes grupos etários, enquanto para câncer de estômago os modelos lineares se apresentaram significativos. Entre as mulheres de 60-69 anos, foi observada diminuição anual média de 0,64 óbito por 100.000 no período de estudo $\left(R^{2}=0,86\right)$, para aquelas de 70-79 anos esse valor correspondeu a 1,40 óbito $\left(\mathrm{R}^{2}=0,86\right)$ e para o grupo de 80 anos e mais, houve, em média, diminuição de 1,78 óbito a cada ano $\left(R^{2}=0,49\right)$. Para câncer de cólon e reto, e de pâncreas o modelo linear foi estatisticamente significativo, mostrando tendência de incremento em todas as faixas etárias. Para câncer de cólon e reto houve, a cada ano, aumento médio de 0,32 óbito por 100.000 mulheres de 60-69 anos $\left(R^{2}=0,59\right)$, de 0,39 óbito em mulheres de $70-79$ anos $\left(\mathrm{R}^{2}=0,29\right)$ e de 1,70 óbito em mulheres de 80 ou mais anos de idade $\left(R^{2}\right.$
= 0,57). O câncer de pâncreas apresentou aumento anual médio de 0,24 óbito por 100.000 mulheres de 60-69 anos $\left(\mathrm{R}^{2}=0,58\right)$, de 0,69 óbito naquelas de 70-79 anos $\left(\mathrm{R}^{2}=0,63\right)$ e de 1,90 óbito em mulheres de 80 ou mais anos de idade $\left(\mathrm{R}^{2}=0,71\right)$.

A evolução da mortalidade por câncer de pulmão, mama e colo de útero na região Sudeste, no período do estudo se encontra na Figura 3. Os modelos lineares mostraram tendência significativa de incremento das taxas de mortalidade por câncer de pulmão e mama em todas as faixas etárias. Para o grupo de 60-69 anos houve, a cada ano, aumento médio de 0,48 óbito por 100.000 mulheres de câncer de pulmão $\left(\mathrm{R}^{2}=0,90\right)$ e de 0,29 óbito por 100.000 mulheres para câncer de mama $\left(\mathrm{R}^{2}=0,36\right)$; na faixa de 70-79 anos, o aumento médio anual foi de 0,88 e 0,74 óbito, respectivamente para câncer de pulmão $\left(\mathrm{R}^{2}=0,80\right)$ e de mama $(0,57)$; entre as mulheres de 80 ou mais anos de idade, a taxa de mortalidade para câncer de pulmão cresceu, em média, 1,53 óbito a cada ano $\left(R^{2}=0,86\right)$, enquanto o câncer de mama teve aumento médio anual de 1,78 óbito por $100.000\left(\mathrm{R}^{2}=0,72\right)$. Para as taxas 
Taxas de mortalidade por câncer de esôfago em

mulheres de 60 ou mais anos de idade

Região Sul, 1980-2005

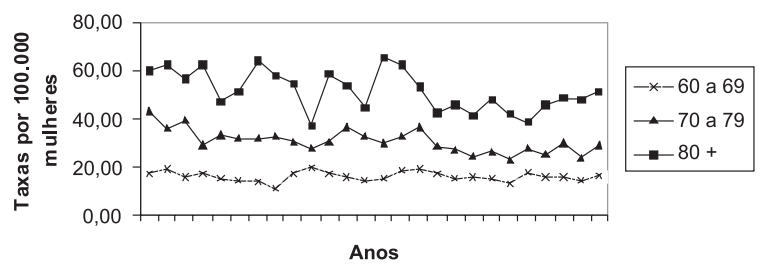

Taxas de mortalidade por câncer de cólon e reto em mulheres de 60 ou mais anos de idade Região Sul, 1980-2005

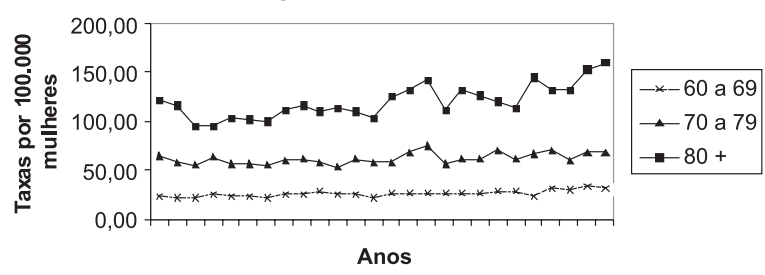

Taxas de mortalidade por câncer de estômago em mulheres de 60 ou mais anos de idade Região Sul, 1980-2005

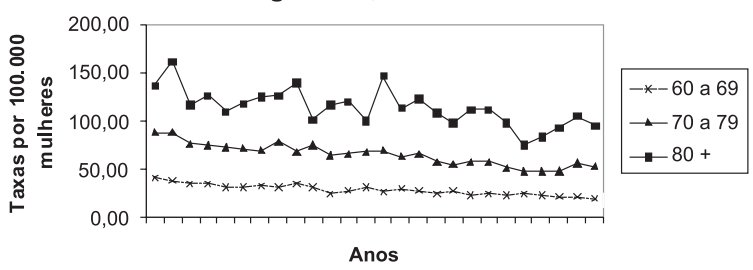

Taxas de mortalidade por câncer de pâncreas em mulheres de 60 ou mais anos de idade Região Sul, 1980-2005

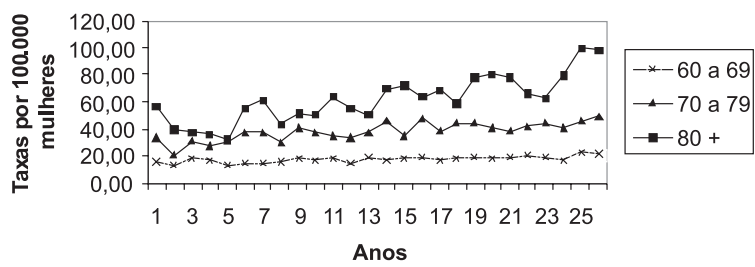

Figura 2 - Taxas de mortalidade por faixa etária (por 100.000) por câncer de esôfago, estômago, cólon e reto e pâncreas em mulheres de 60 ou mais anos de idade na região Sul do Brasil no período 1980-2005.

Figure 2 - Mortality rates per age group (per 100,.000) for esophageal, gastric, colon/rectal, and pancreatic cancers in women aged 60 or more in the South region of Brazil in the 1980-2005 period.

de mortalidade por câncer de colo de útero nenhum dos modelos testados apresentou significância estatística.

A evolução da mortalidade por essas localizações de câncer na região Sul encontra-se na Figura 4. Novamente os modelos lineares mostraram-se adequados e apresentaram significância estatística nas três faixas etárias analisadas, em relação aos tumores de pulmão e mama. No grupo etário de 60-69 anos, a cada ano houve aumento de 1,21 óbito por 100.000 na taxa de mortalidade por câncer de pulmão $\left(\mathrm{R}^{2}=0,92\right)$, e de 0,42 óbito por 100.000 na taxa de mortalidade por câncer de mama $\left(R^{2}=0,42\right)$. Para o grupo de 70-79 anos, a taxa de mortalidade por câncer de pulmão aumentou, em média, 1,88 óbito por 100.000 ao ano $\left(\mathrm{R}^{2}=0,89\right)$ e a taxa de câncer de mama, 1,17 óbito $\left(R^{2}=0,75\right)$, enquanto no grupo de 80 anos e mais esse aumento correspondeu a 2,45 óbitos $\left(\mathrm{R}^{2}=0,82\right)$ para câncer de pulmão e a 2,46 óbitos/ 100.000 para câncer de mama $\left(R^{2}=0,70\right)$. Para câncer de colo de útero, só foi possível identificar um modelo com significância estatística para o grupo de 60-69 anos. Nessa faixa etária, o modelo linear mostrou tendência de crescimento das taxas de mortalidade, com aumento de 0,17 óbito por 100.000 mulheres a cada ano $\left(\mathrm{R}^{2}=0,36\right)$.

\section{Discussão}

Em nosso estudo observamos certa heterogeneidade no padrão de mortalidade por câncer das mulheres idosas, segundo faixa etária e região. Eventos ou estilos de vida adotados em idades mais jovens podem ser determinantes das tendências de mortalidade de populações idosas ${ }^{7}$, sendo possível que diferenças regionais e entre as gerações tenham contribuído para as diferenças observadas. Entretanto, estudos sobre fatores de risco e proteção para câncer são escassos no Brasil, dificultando o conhecimento de sua distribuição na população feminina e suas possíveis relações com as tendências de mortalidade. É importante considerar, também, que ocorreu redução do percentual de óbitos por causas mal definidas no período de estudo, nas três faixas etárias e em ambas as regiões. Essa redução, que foi mais acentuada na região Sul, poderia ter contribuído para uma superestimação dos 
incrementos observados, devido à inclusão de óbitos por câncer, que anteriormente não tinham a causa básica esclarecida.

Wünsch e Moncau ${ }^{5}$, analisando uma série histórica que vai de 1980 a 1995, observaram redução na mortalidade por câncer de esôfago e de estômago para o conjunto da população feminina das regiões Sul e Sudeste, e Hallal et $\mathrm{al}^{8}$, no Rio Grande do Sul, verificaram tendência de decréscimo da

\section{Taxas de mortalidade por câncer de pulmão em mulheres de 60 ou mais anos de idade Região Sudeste, 1980-2005}

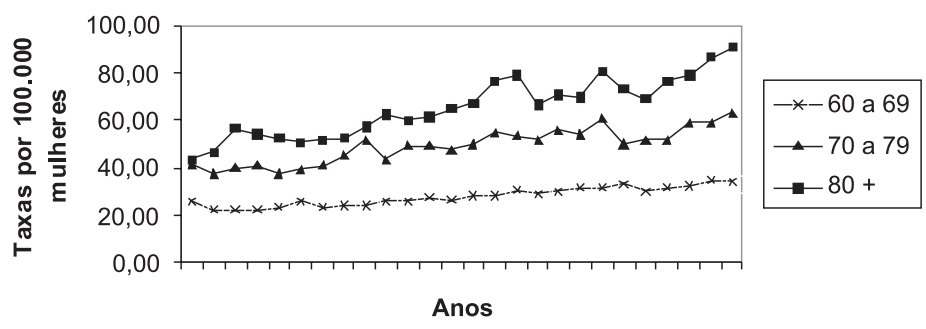

Taxas de mortalidade por câncer de mama em mulheres de 60 ou mais anos de idade Região Sudeste, 1980-2005

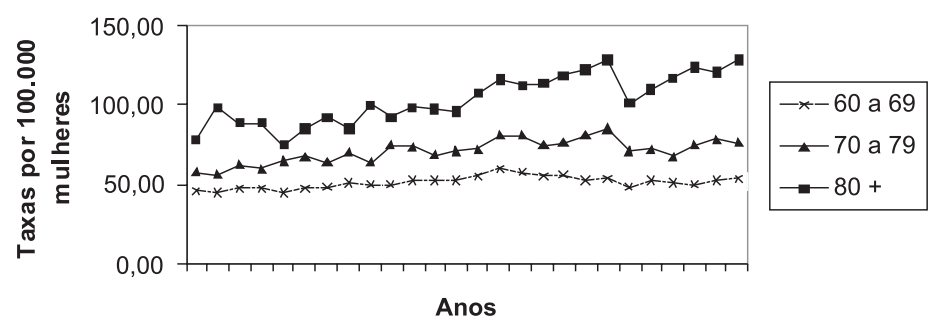

Taxas de mortalidade por câncer de colo de útero em mulheres de 60 ou mais anos de idade Região Sudeste, 1980-2005

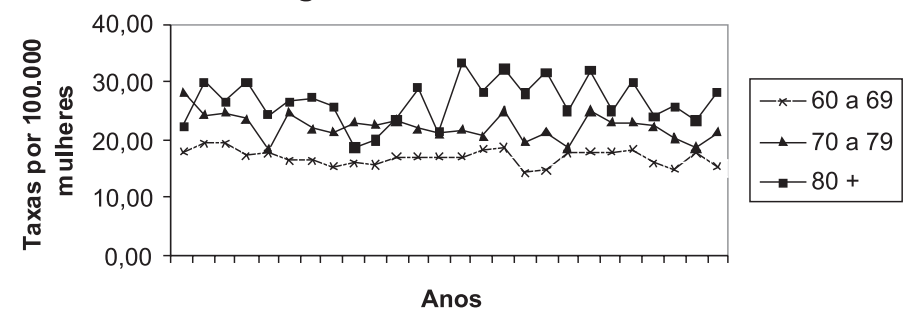

Figura 3 - Taxas de mortalidade por faixa etária (por 100.000) por câncer de pulmão, mama e colo de útero em mulheres de 60 ou mais anos de idade na região Sudeste do Brasil no período 1980-2005.

Figure 3 - Mortality rates per age group (per 100,.000) for lung, breast, and uterine cervix cancers in women aged 60 or more in the Southeast region of Brazil in the 1980-2005 period. mortalidade por câncer de estômago, porém não para câncer de esôfago. Embora nosso estudo tenha sido exclusivamente com a população feminina idosa e os períodos de tempo analisados sejam distintos, os resultados observados foram similares, apontando para a continuidade desse declínio.

O declínio da mortalidade por essas duas localizações de câncer têm sido observado em diferentes países do mundo ${ }^{9-12} \mathrm{e}$, no caso do estômago, é atribuída à diminuição da incidência, relacionada à melhor conservação dos alimentos, à dieta mais variada $\mathrm{e}$ afluente e à redução da prevalência da infecção por Helicobacter pylori ${ }^{10}$, já que não se verificaram modificações marcantes no seu prognóstico ${ }^{13,14}$. A dieta também tem sido considerada como um fator relacionado ao desenvolvimento de câncer de esôfago, especialmente entre populações com baixa prevalência de tabagismo ${ }^{13}$.

O incremento das taxas de mortalidade por câncer de cólon e reto foi observado em diferentes países da América ${ }^{10}$ e tem sido atribuído ao consumo excessivo de carnes vermelhas e de gorduras, ao baixo consumo de frutas, verduras e fibras, à obesidade e ao sedentarismo ${ }^{15}$. Em nosso estudo, verificamos tendência significativa de aumento da mortalidade por câncer de cólon e reto e pâncreas em todas as faixas etárias nas duas regiões. Wünsch e Moncau ${ }^{5}$ observaram estabilidade das taxas de mortalidade por câncer de pâncreas e variação percentual positiva das taxas de mortalidade por câncer de cólon e reto.

Estudos na população brasileira indicam que a prevalência de Helicobacter pylori variou entre 66 e 34\%, dependendo da idade e da população estudada ${ }^{16,17}$. Em relação à dieta, ocorreram modificações no padrão alimentar nas duas regiões estudadas, com melhora do aporte protéico e de outros nutrientes essenciais, intensificação do consumo de produtos de origem animal, estabilização no consumo de cereais e derivados e aumento do conteúdo lipídico da dieta ${ }^{18,19}$. Um estudo em Minas Gerais mostrou que um terço dos indivíduos adultos estudados excediam o limite máximo 
recomendado para consumo de lipídios ${ }^{20}$, enquanto em outro, na cidade de São Paulo, foi observado que cerca de $20 \%$ das mulheres de 50-59 anos estudadas apresentavam colesterol total ou triglicérides acima dos limites considerados adequados ${ }^{21}$.

A redução ou estagnação do consumo de cereais, leguminosas, verduras, legumes e frutas nas regiões Sudeste e Sul vem ocorrendo desde a década de $60^{18,19}$. Estudos mais recentes mostram consumo de frutas e hortaliças bastante similar entre mulheres de 50 anos e mais nas capitais das duas regiões ${ }^{22.23}$. A prevalência de consumo de frutas diminui com a idade, mas não o consumo de hortaliças, considerando o conjunto das capitais brasileiras ${ }^{23}$. Embora existam indícios de diferenciais de consumo entre as gerações, os dados disponíveis não permitem averiguar se isso ocorreu de forma similar no Sul e no Sudeste. Najas et al. ${ }^{24}$, em um estudo realizado somente com indivíduos idosos residentes em São Paulo, observaram que $85 \%$ destes tinham hábito de consumir frutas, verduras e legumes.

Um estudo recente mostrou prevalência de obesidade similar nas regiões Sudeste e Sul e percentuais bastante próximos de mulheres consideradas como irregularmente ativas ${ }^{22}$. Nas capitais brasileiras, são fisicamente inativas $23,0 \%$ das mulheres entre 55 a 64 anos e 50,3\% daquelas com 65 anos ou mais ${ }^{23}$. Embora não se disponha de dados para avaliar a evolução da obesidade em mulheres idosas, a maior prevalência de excesso de peso em idades avançadas e o aumento acentuado do sedentarismo poderiam ter sido fatores contribuintes para o incremento observado das taxas de mortalidade por câncer de cólon.

O aumento da mortalidade por câncer de pulmão no sexo feminino vem ocorrendo praticamente no mundo todo ${ }^{9,10,25}$. Hallal et al. ${ }^{8}$ verificaram tendência de incremento das taxas de mortalidade por esse câncer nas mulheres do Rio Grande do Sul, e Wünsch e Moncau ${ }^{5}$ observaram aumento percentual nas taxas do sexo feminino nas duas regiões. Lima-Costa et al. ${ }^{3}$ observaram aumento da taxa de mortalidade por esse câncer em mulheres idosas em ambas as regiões. Os diferentes padrões de mortalidade observados para esse câncer entre o Sul e o Sudeste possivelmente refletem diferenças na prevalência de tabagismo entre as mulheres. Em 1987, a prevalência de tabagismo em mulheres de 15-59 anos em São Paulo e em Porto Alegre eram bastante similares ${ }^{26,27} \mathrm{e}$, embora tenha sido observada redução da prevalência de tabagismo na população feminina de 15 anos ou mais em todas as capitais das duas regiões, os percentuais mais altos de fumantes

\section{Taxas de mortalidade por câncer de pulmão em mulheres de 60 ou mais anos de idade Região Sul, 1980-2005}

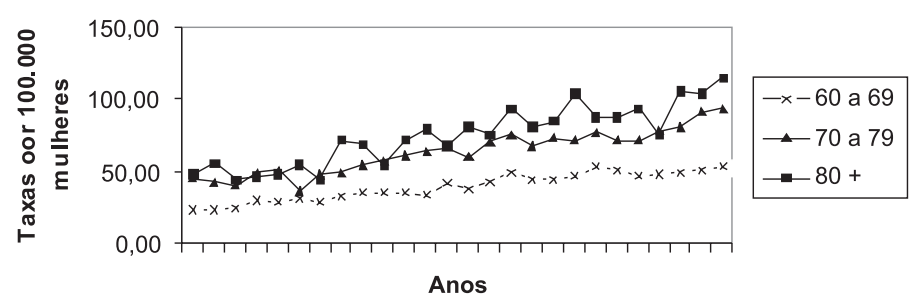

Taxas de mortalidade por câncer de mama em mulheres de 60 anos ou mais de idade Região Sul, 1980-2005

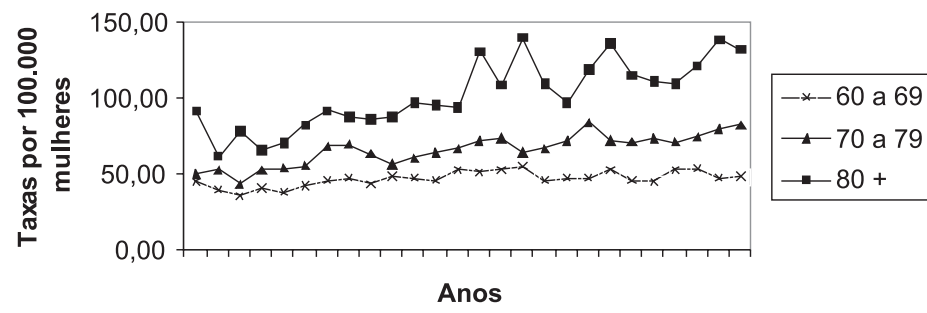

Taxas de mortalidade por câncer de colo de útero em mulheres de 60 ou mais anos de idade Região Sul, 1980-2005

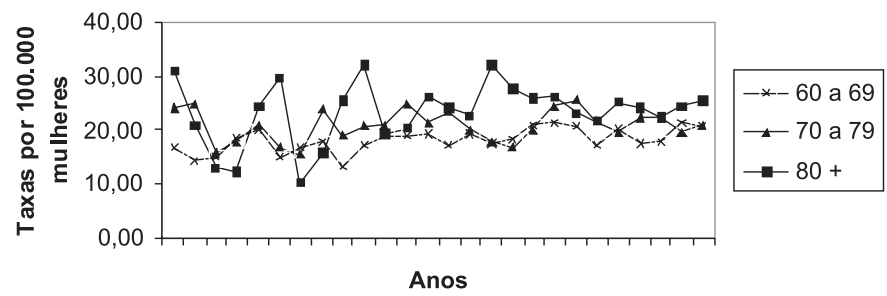

Figura 4 - Taxas de mortalidade por faixa etária (por 100.000) por câncer de pulmão, mama e colo de útero em mulheres de 60 ou mais anos de idade na região Sul do Brasil no período 1980-2005.

Figure 4 - Mortality rates per age group (per 100,000) for lung, breast, and uterine cervix cancers in women aged 60 or more in the South region of Brazil in the 1980-2005 period. 
foram registrados no Sul ${ }^{22}$. Em Minas Gerais, em estudo com indivíduos idosos, observouse associação negativa entre hábito de fumar e idade no sexo feminino ${ }^{28}$.

Em anos recentes, foram observadas tendências de declínio das taxas de mortalidade por câncer de mama em mulheres européias e nos Estados Unidos ${ }^{9,11}$. Naquele país, entretanto, o percentual médio de redução anual, no período 1998-2002, foi menor nas mulheres de 50 anos ou mais, em comparação com as mais jovens ${ }^{29}$. Em nosso estudo, verificamos tendência de incremento das taxas de mortalidade por esse câncer nas três faixas etárias, em ambas as regiões, com incremento médio anual mais alto na região Sul, à semelhança dos achados de Wünsch e Moncau ${ }^{5}$. Zago et al. ${ }^{30}$ observaram tendência semelhante para mulheres de 20 a 59 anos de idade em municípios da Baixada Santista, enquanto Gonçalves et al. ${ }^{31}$ observaram tendência similar nos três estados da região Sul. Diferenças em relação a hábitos de vida, história reprodutiva e idade média da menarca e da menopausa, assim como nas práticas de controle do câncer de mama entre as regiões e entre coortes de nascimento poderiam ser parcialmente responsáveis pelas variações observadas. O percentual de mulheres que haviam realizado mamografia nos dois últimos anos que antecederam a realização de um inquérito variou entre 49,6 e 76,5\% nas capitais das regiões Sul e Sudeste ${ }^{22}$, evidenciando variabilidade dessa prática.

Em relação ao câncer de colo de útero, estudos epidemiológicos têm apontado a necessidade da análise conjunta da mortalidade por essa localização e pela porção não especificada do útero, já que grande parte dos óbitos assim classificados pode ter como sítio de origem o colo uterino ${ }^{8,32}$ Entretanto, a CID-BR não disponibiliza informações relativas ao número de óbitos por porção não especificada do útero isoladamente, mas apenas agrupados com corpo do útero, impossibilitando esse tipo de análise em nosso estudo. Assim, é pos- sível que nossos resultados estejam representando apenas uma parcela do conjunto de óbitos por câncer de colo uterino, o que não permitiu a adequada caracterização do padrão de mortalidade das mulheres idosas. Analisando a mortalidade por esse câncer no Rio Grande do Sul, Kalakun e Bozzeti $^{33}$ observaram tendência linear de incremento das taxas, enquanto Hallal et al. ${ }^{8}$, que incluiram no numerador de suas taxas, além dos óbitos por câncer de colo, aqueles de porção não especificada do útero, observaram, para o mesmo estado, tendência de estabilidade..

A utilização de dados de mortalidade tem como vantagem a maior estabilidade em relação à qualidade, a abrangência e a disponibilidade para maiores períodos de tempo, embora mudanças na assistência médica e no acesso ao diagnóstico e tratamento de câncer ao longo do tempo possam afetar análises que tenham esses dados como base. Por outro lado, a cobertura e a qualidade do Sistema de Informações sobre Mortalidade é reconhecidamente melhor nas regiões Sul e Sudeste ${ }^{5}$, e estudos epidemiológicos brasileiros têm demonstrado a fidedignidade da informação sobre o câncer como causa básica de morte ${ }^{32,34}$. A estimação de tendências através de modelos de regressão polinomial apresenta vantagens, entre elas a facilidade de elaboração e interpretação e o grande poder estatístico ${ }^{35}$. Entretanto, também tem limitações, como a possiblidade de presença de multicolinearidade $^{36}$. Buscando evitá-la, utilizamos o ponto médio da série histórica na construção dos modelos analisados ${ }^{35}$.

Na literatura, são escassos os estudos que analisam padrões de mortalidade por câncer em idosos. Nesse sentido, o presente estudo buscou contribuir para o maior conhecimento das condições de saúde da população idosa feminina em nosso país, apontando para a importância do câncer como problema de saúde pública nesse grupo populacional. 


\section{Referências}

1. Organização Mundial de Saúde (OMS). Temas de salud. http://www.who.int/es/ [Acessado em setembro 2006].

2. Camarano AA. Brazilian population ageing: A demographic contribution. IPEA Working Paper No. 858; 2002.

3. Lima-Costa MF, Peixoto SV, Giatti L. Tendências da mortalidade entre idosos brasileiros (1980-2000). Epidemiologia e Serviços de Saúde 2004; 13(4): 217-28.

4. Vercelli M, Lillni R, Capocaccia R et al. Cancer survival in the elderly: Effects of socio-economic factors and health care system features (ELDCARE project). European Journal of Cancer 2006; 42: 234-42.

5. Wünsch Filho V, Moncuau JE. Mortalidade por câncer no Brasil 1980-1995: Padrões regionais e tendências temporais.Revista da Associação Médica Brasileira 2002; 48(3): 250-7.

6. Ministério da Saúde, Brasil. Informações de Saúde. http://w3.datasus.gov.br/datasus/datasus.php [Acessado em agosto de 2006].

7. Janssen F, Mackenbach JP, Kinst AE. Trends in old-age mortality in seven European countries, 1950-1999. J Clin Epidemiol 2004; 57: 203-16.

8. Hallal ALC, Gotlieb SL, Latorre MRDO. Evolução da mortalidade por neoplasias malignas no Rio Grande do Sul, 1979-1995. Rev Bras Epidemiol 2001; 4(3): 168-75.

9. Edwards BK, Brown ML, Wingo, PA et al. Annual report to the Nation on the status of cancer, 1975-2002, featuring population-based trends in cancer treatment. $J$ Nation Cancer Instit 2005; 97(19):1407-27.

10. Bosetti C, Malvezzi M, Chatenoud L et al. Trends in cancer mortality in the Americas, 1970-2000. Ann Oncol 2005; 16: 489-501.

11. Boyle P, Ferlay J.. Cancer incidence and mortality in Europe, 2004. Ann Oncol 2005; 16: 481-8.

12. Inque M, Tsugane S. Epidemiology of gastric cancer in Japan. Postgrad Med J 2005; 81: 419-24.

13. Bouvier AM, Binquet C, Gagnaire A et al. Management and prognosis of esophageal cancers : Has progress been made? Eur J Cancer 2006; 42: 228-33.

14. Crew KD, Neuget AI. Epidemiology of gastric cancer. World J Gastroenterol 2006; 12(3): 354-62.

15. Organização Mundial de Saúde (OMS). Diet, nutrition and the prevention of chronic diseases. Report of a joint WHO/FAO Expert Consultation: Geneva; 2003.

16. Rocha GA, Queiroz DM, Mendes EN, Oliveira AM, Moura SB, SilvaRJ. Source of Helicobacter pylori infection: Studies in abattoir workers and pigs. Am J Gastroenterol 1992; 87: 1525,.
17. Oliveira AMR, Queiroz, DMM, Rocha GA, Mendes EN. Seroprevalence of Helicobacter pylori infection in children of low socioeconomic level in Belo Horizonte, Brazil. Am J Gastroenterol 1994; 89: 2201-04.

18. Mondini L, Monteiro CA. Mudanças no padrão de alimentação da população urbana brasileira (1962-1988). Rev Saúde Pública 1994; 28(6): 433-9.

19. Monteiro CA, Mondini L, Costa RBL.. Mudanças na composição e adequação nutricional da dieta familiar nas áreas metropolitanas do Brasil (1988-1996). Rev Saúde Pública 2000; 34(3): 251-8.

20. Bonomo E, Caiaffa WT, Lopes ACS, Lima-Costa MF. Consumo alimentar da população adulta segundo perfil socioeconômico e demográfico: Projeto Bambuí. Cad Saúde Pública 2003; 19(5): 1461-71.

21. Marcopito LF, Rodrigues SSF, Pacheco MA, Shirassu M, Goldfeder AJ, Moraes MA. Prevalência de alguns fatores de risco para doenças crônicas na cidade de São Paulo. Rev Saúde Pública 2005; 39(5): 738-45.

22. Ministério da Saúde. Inquérito domiciliar sobre comportamentos de risco e morbidade referida de doenças e agravos não transmissíveis 2002-2003. Brasília, DF; 2004.

23. Ministério da Saúde. Vigitel Brasil 2006 - Vigilância de fatores de risco e proteção para doenças crônicas por inquérito telefônico. Brasília, DF; 2007.

24. Najas MS, Andreazza R, Souza ALM, Sachs A, Guedes ACB, Sampaio LR, Ramos LR, Tudisco ES. Padrão alimentar de idosos de diferentes estratos socioeconômicos residentes em localidade urbana da região sudeste, Brasil. Rev Saúde Pública 1994; 28(3): 187-91.

25. Liaw YP, Huang YC, Lien GW. Patterns of lung cancer mortality in 23 countries: Application of the age-periodcohort model. BMC Public Health 2005; 5(1): 22.

26. Rego RA, Berardo FAN, Rodrigues SSR, Oliveira ZMA, Oliveira MB, Vasconcellos C, Aventurato LVO, Moncau JEC, Ramos LR. Fatores de risco para doenças crônicas não transmissíveis: inquérito domiciliar no município de São Paulo, SP (Brasil), Metodologia e resultados preliminares. Rev Saúde Pública 1990; 24(4): 277-85.

27. Dundan BB, Schmidt ML, Polanczyk CA, Homrich CS, Rosa RS, Achutti AC. Fatores de risco para doenças não transmissíveis em área metropolitana na região sul do Brasil, Prevalência e simultaneidade. Rev Saúde Pública 1993; 27(1): 143-8.

28. Peixoto SV, Firmo JOA, Lima-Costa MF.Condições de saúde e tabagismo entre idosos residentes em duas comunidades brasileiras (Projetos Bambuí e Belo Horizonte). Cad Saúde Pública 2006; 22(9): 1925-34.

29. Smigal C, Jemal A, Ward E et al. Trends in breast cancer by race and ethnicity: Update 2006. Cancer J Clinicians 2006; 56(3): 168-83. 
30. Zago A,Pereira, LAA, Braga ALF et al. Mortalidade por câncer de mama em mulheres na Baixada Santista, 1980 a 1999. Rev Saúde Pública 2005; 39(4): 641-5.

31. Gonçalves ATC, Jobim PFC, Vanacor R, Nunes LN, Albuquerque, IM, Bozetti MC. Câncer de mama: mortalidade crescente na Região Sul do Brasil entre 1980 e 2002. Cad Saúde Pública 2007; 23(8): 1785-90.

32. Nunes J, Koifman R, Mattos IE et al. Confiabilidade e validade das declarações de óbitos por câncer de útero no Município de Belém Pará. Cad Saúde Pública 2004; 20(5): 1262-8.

33. Kalakun L, Bozzetti MC. Evolution of uterine cervical cancer mortality from 1979 to 1998 in the State of Rio Grande do Sul, Brazil. Cad Saúde Pública 2005; 21(1): 299-309.
34. Queiroz RC, Mattos IE, Monteiro GTR et al. Confiabilidade e validade das declarações de óbito por câncer de boca no Município do Rio de Janeiro. Cad Saúde Pública 2003; 19(6): 1645-54.

35. Latorre MRDO, Cardoso MRA. Análise de séries temporais em epidemiologia: uma introdução sobre os aspectos metodológicos. Rev Bras Epidemiol 2001; 4(3): 145-52.

36. Neter J, Kutner MH, Wasserman W. Building the Regression Model III: Remedial Measures and Validation. In: Applied linear statistical models. 4 th edition. WCB McGraw-Hill: Boston; 1996.

Recebido em: 03/04/07 Versão final reapresentada em: 02/04/08 Aprovado em: 08/04/08 